\title{
苯并富烯合成方法研究进展
}

\author{
史传星 $^{a, b}$ 冯陈国 $b, c$ 陈雅丽*,a 张曙盛 $*, b, c$ 林国强 ${ }^{b}$ \\ $\left({ }^{a}\right.$ 上海大学理学院 上海 200444)) \\ $\left({ }^{b}\right.$ 中国科学院上海有机化学研究所 天然产物有机合成化学重点实验室 上海 200032) \\ ( ${ }^{c}$ 上海中医药大学 创新中药研究院 上海 201203)
}

\begin{abstract}
摘要 苯并富烯结构存在于许多天然分子和生物活性分子中, 同时此类分子常常作为重要的合成砌块运用于材料科学 和金属有机化学等领域. 对苯并富烯的合成已进行了大量研究，尤其是近二十年的快速发展，已经发展了系列高效的 合成方法. 依据关键骨架构建反应引发机制的不同, 这些方法大致可分为五大类: 热引发或者光催化的双自由基机理 的环化反应、过渡金属催化的串联环化反应、亲核或亲电试剂进攻引发的环化反应、自由基引发的环化反应以及酸促 进的环化反应. 本文将根据上述不同反应类型综述近些年苯富烯合成的研究进展.
\end{abstract}

关键词＼cjkstart苯并富烯; 过渡金属催化; 双自由基; $\mathrm{C}-\mathrm{H}$ 键活化; 环化反应

\section{Recent Advancement in Benzofulvene Synthesis}

\author{
Shi, Chuanxing ${ }^{a, b}$ \\ Feng, Chenguo ${ }^{b, c}$ \\ Chen, Yali*,a \\ Zhang, Shusheng ${ }^{*, b, c}$ \\ Lin, Guoqiang ${ }^{b}$ \\ ( ${ }^{a}$ College of Sciences, Shanghai University, Shanghai 200444) \\ $\left({ }^{b}\right.$ CAS Key Laboratory of Synthetic Chemistry of Natural Substances, Shanghai Institute of Organic Chemistry, \\ Chinese Academy of Sciences, Shanghai 200032) \\ ( ${ }^{c}$ Innovation Research Instutute of Tranditional Chinese Medicine, Shanghai University of Tranditional \\ Chinese Medicine, Shanghai 201203)
}

\begin{abstract}
Benzofulvenes were widely found in natural products and bioactive molecules, and also served as important building blocks in material science and transition-metal chemistry. Great efforts have been devoted to the efficient synthesis of these interesting molecules, and rapid advancement has been made in the past two decades. According to the types of the initiation of the reaction, these methods can roughly be classified into five categories: thermal or photochemical cyclization of enyne-allenes or enediynes, transition metal-catalyzed sequential cyclization reaction, electrophilic or nucleophilic attack initiated cyclization, radical initiated cyclization and acid promoted cyclization. This review describes the important synthetic methods of benzofulvenes according to their reaction types.

Keywords benzofulvenes; transition metal catalysis; radical; $\mathrm{C}-\mathrm{H}$ bond activation; cyclization reaction
\end{abstract}

富烯 ${ }^{[1]}$ 是一类具有环外碳-碳双键的环戊二烯骨架 的分子(Figure 1). 早期, 其特指 5-亚甲基-1,3-环戊二烯 (1a). 随着对非苯芳香现象的认识的深入, 大量结构类 似于富烯的分子被发现, 富烯的概念得到了进一步的拓 展, 泛指含有奇数个原子的共轭环与一个环外双键形成 的具有交叉共轭(cross-conjugation)结构的分子(1b). 由 于共振的存在, 环外双键的电子与环上共轭体系的 $\pi$ 电 子发生部分转移以满足分子中环状结构单元的芳香性.

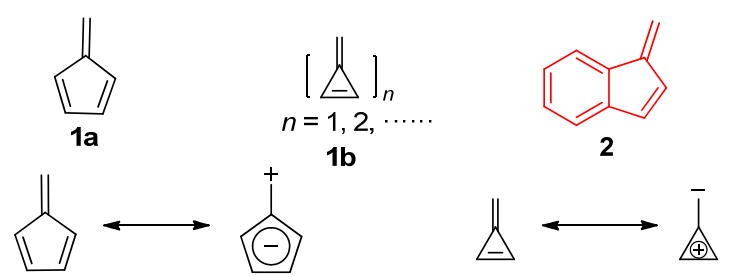

图 1 富烯及其共振结构

Figure 1 Fulvene and its resonance structure

\footnotetext{
* Corresponding authors. E-mail: ylchen@staff.shu.edu.cn; zhangss@sioc.ac.cn

Received October 25, 2019; revised December 17, 2019; published online January 3, 2020.

Project supported by the National Natural Science Foundation of China (Nos. 21572253, 21772216), the Strategic Priority Research Program of the Chinese Academy of Sciences (No. XDB 20020100), and the Key Research Program of Frontier Science (No. QYZDY-SSWSLH026).

国家自然科学基金(Nos. 221572253, 21772216)、中国科学院战略性先导科技专项(No. XDB 20020100)、中国科学院前沿科学重点研究(No. QYZDYSSWSLH026)资助项目.
} 
与此同时, 由于环外双键 $\pi$ 电子部分转移至环上或环上 的 $\pi$ 电子部分转移至外双键上(图 1), 使得分子内存在一 个较大的偶极矩 ${ }^{[2]}$, 表现出独特的物理和化学性质.

苯并富烯 2 及其衍生物则是富烯结构中非常重要的 一类化合物. 苯并富烯及其衍生物含有一个狮骨架, 又 称为 1 -亚甲基茛. 狮是许多药物分子和天然产物的基本 骨架 ${ }^{[3]}$, 也是众多材料分子的重要前体 ${ }^{[4]}$. 与狮骨架相 比, 苯并富烯骨架含有一个环外的双键, 容易被官能团 化. 苯并富烯不仅是一些活性生物分子 ${ }^{[5]}$ 和天然产物的 核心结构骨架, 同时也是一类重要的合成中间体 ${ }^{[6]}$ (图 2). 2009 年, Sarpong 课题组 ${ }^{[6 b]}$ 报道了一例利用苯并富烯 骨架中间体合成二聚白黎芦醇的方法, 由甲氧基取代苯 基苯并富烯经过一系列反应可得到两种具有活性的天 然产物 quadrangularin A 和 parthenocissin A. 而非固醇类 抗炎药物舒林酸(4)和舒林酸砜(5)的核心骨架也是含有 氟取代的苯并富烯结构 ${ }^{[5 b]}$. 苯并富烯也被应用于高分 子功能材料研究中, 如具有此类新颖结构的高聚物 $\mathbf{6}$, 可以用于光电材料并且前景广阔 ${ }^{[7]}$. 通过改变官能团可 以改变聚合物的性质. 苯并富烯及其衍生物含有一个环 戊二烯的骨架, 可用于制备后茂金属催化剂 ${ }^{[8]}$ (Postmetallocene catalysts), 如金属锆络合物 7, 应用于不对 称的烯烃聚合反应 ${ }^{[9]}$ 、Diels-Alder 反应等 ${ }^{[10]}$.
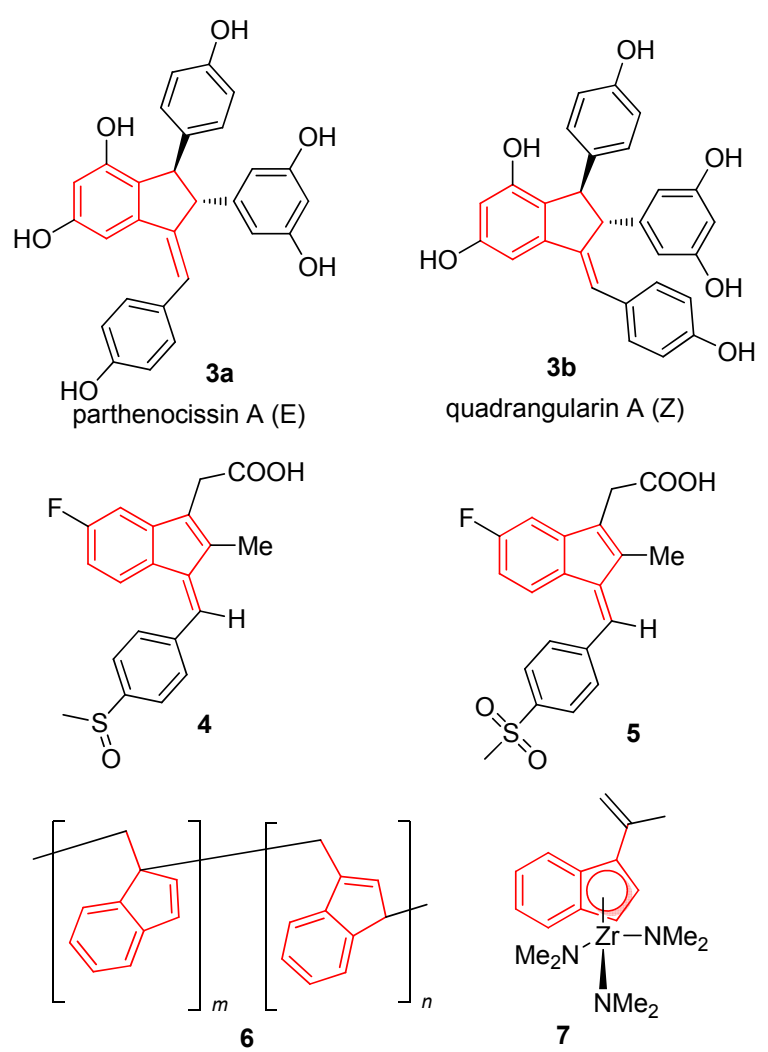

图 2 含苯并富烯骨架的生物活性分子和功能材料

Figure 2 Bioactive molecules and materials containing benzofulvene moiety
狮与醛或酮在碱催化作用下的缩合反应是合成苯 并富烯的传统方法. 首先, 狮 8 在碱的作用下, 失去质 子转化为狮负离子 9 , 然后进攻醛/酮的羰基, 脱水形成 苯并富烯 10 (Scheme 1). 这一方法官能团兼容性存在局 限, 难以应用于不同取代基结构的苯并富烯合成 ${ }^{[11]}$.

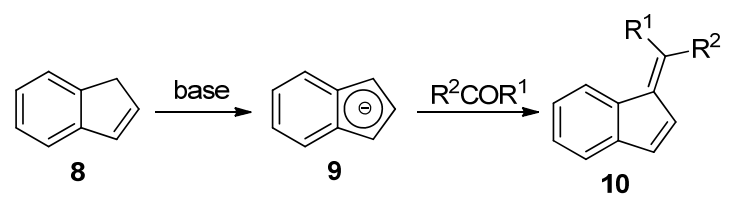

图式 1 苯并富烯合成的传统方法

Scheme 1 Traditional method of benzofulvene synthesis

苯并富烯及其衍生物的应用广泛，其合成方法也得 到了迅速的发展. 按照关键的环化反应的不同引发方 式，构建苯并富烯骨架的主要方法可以大致分为五大 类: 光催化或者热引发的联烯一烯炔或者烯二炔环化反 应、过渡金属催化的串联环化反应、亲核或亲电试剂进 攻引发的环化反应、自由基引发的环化反应，以及路易 斯酸促进的环化反应. 本文将按照上述反应分类，对苯 并富烯类化合物的合成方法进行总结.

\section{1 光或者热引发的联烯一烯炔或者烯二炔环化 反应}

Bergman 环芳香化及其相关反应是有机化学、药物 化学和材料化学领域里的一类非常重要的反应 ${ }^{[12]}$. 这 类反应以烯二炔类和烯炔-联烯类化合物为底物, 常常 会经历一个双自由基中间体 ${ }^{[12]}$. Bergman 环芳香化反应 也是烯二炔类抗生素具有高效抗肿瘤活性的决定性因 素之一. 烯二炔类抗生素结构中含有双烯双炔共轭大 环，通过 Bergman 反应形成苯基双自由基活性中间体， 直接破坏肿瘤细胞 DNA 链, 最终引发肿瘤细胞调亡.

Bergman 环化反应发生在烯二炔的 $C^{1}-C^{6}$ 位，而 Myers-Saito 环化反应 ${ }^{[13]}$ 发生在烯炔-联烯的 $C^{2}-C^{7}$ 位， 这两类反应都是经历双自由基的中间体，最终形成苯环 (Scheme 2). 从反应机理上来看, 这两种底物形成富烯

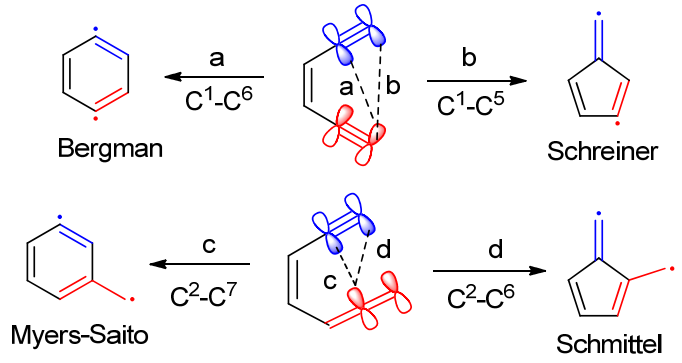

图式 2 烯二炔和烯炔-联烯类底物环芳香化反应的几种模式 Scheme 2 Cycloaromatization patterns of enediyne and enyneallene substrates 
的可能性同样也是存在的. 2001 年 Peter 等 ${ }^{[14]}$ 对于此类 环化反应进行了细致的理论计算. 结果表明上述的 $C^{1}-C^{5}$ 环化反应相比 $C^{1}-C^{6}$ 和 $C^{2}-C^{7}$ 环化反应确实需要更 高的反应活化能, 因而更容易生成苯环. 但是芳环体系 的存在, 以及大位阻末端取代基的应用, 可以促进 $\mathrm{C}^{1}-\mathrm{C}^{5}$ 环化反应. 如果烯二炔末端含有吸电子取代基, 则可以使环化反应所需要的能量进一步降低.

1995 年, Schmittel 等 ${ }^{[15]}$ 研究苯并烯炔-联烯类底物 11 的 $\mathrm{C}^{2}-\mathrm{C}^{6}$ 环化反应时, 发现含有特殊取代基的烯炔联烯类底物 12 在 $50{ }^{\circ} \mathrm{C}$ 时发生 $\mathrm{C}^{2}-\mathrm{C}^{7}$ 的常规 Myers-Saito 环化形成苯环, 当温度升高至 $84{ }^{\circ} \mathrm{C}$ 时, 可以发生 $\mathrm{C}^{2}-\mathrm{C}^{6}$ 的环化反应, 以中等的收率得到了苯并富烯骨架的产物 (Scheme 3a). 虽然原料 12 可由烯炔 11 经过简单的转化 而得, 但是利用这种方法进行合成, 其底物适用性仍然 比较局限 ${ }^{[16]}$.

2002 年, Serguei 课题组 ${ }^{[17]}$ 报道了一例光催化的 1 , 2-二乙炔基苯 14 的 $C^{1}-C^{5}$ 的环化反应(Scheme $3 b$ ). 由于
Peter 预测末端吸电子基团取代的环化反应的能垒较低, 并且实验进行了验证. 所以作者合成了炔基末端是强吸 电子基团的底物 14, 尝试在光照的条件下合成苯并富 烯, 但是未能成功, 以较低收率得到了 $\mathrm{C}^{1}-\mathrm{C}^{5}$ 环化的茚 的产物 15, 16. 实验证明, 仅利用光照, 由 1,2-二乙炔基 苯合成苯并富烯比较困难.

2006 年 Schmittel 课题组 ${ }^{[18]}$ 报道了一例苯并烯炔联烯 17, 通过热引发生成苯并富烯衍生物的方法. 他们 在分子结构上引入自由基时钟模块(Radical clock)环丙 烷，得到了环丙烷开环产物，证明了这个反应的自由基 历程. 这例反应伴有副产物 20 和 21 的产生, 反应产率 比较低(Scheme 4a).

同年, Pascal 课题组 ${ }^{[19]}$ 报道了一例热引发 1,2-二苯 基乙炔构建苯并富烯的反应(Scheme 4b), 这个反应与 传统的利用这类底物构建苯并富烯的方法类似，会发生 $C^{1}-C^{6}, C^{1}-C^{5}$ 两种环化反应(Scheme 2). 他们课题组也

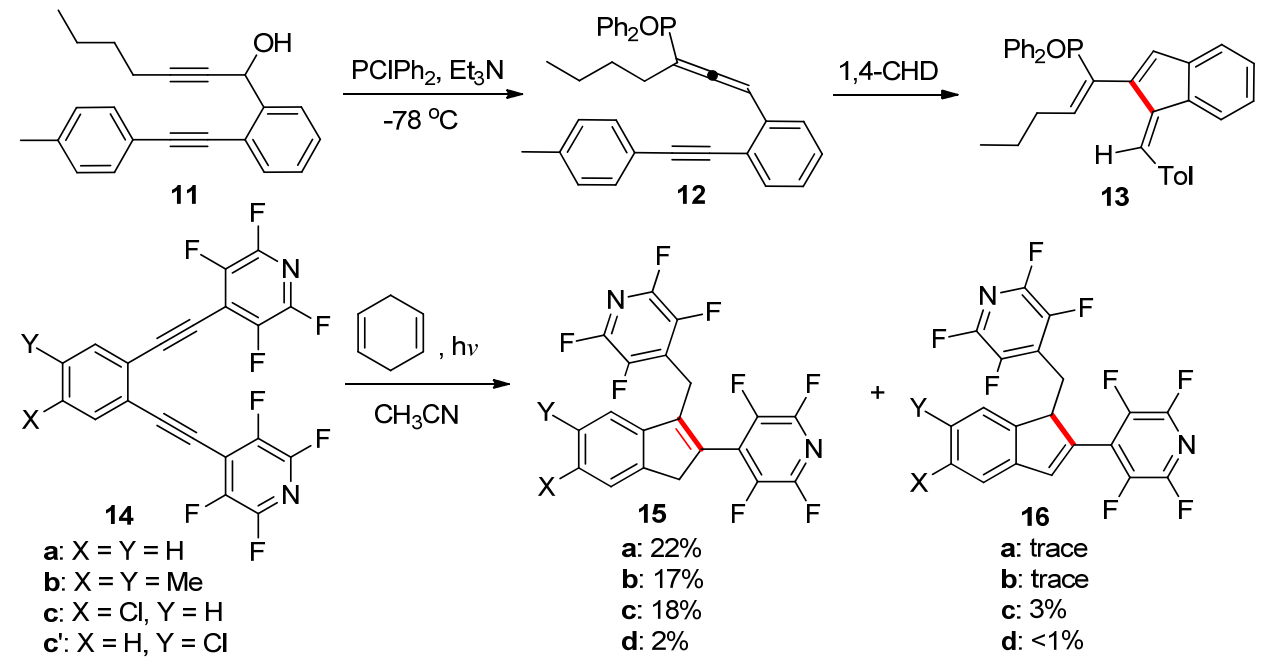

图式 3 光或热引发联烯一烯炔环化构建苯并富烯

Scheme 3 Synthesis of benzofulvenes by photo/thermal cyclization of enyne-allenes

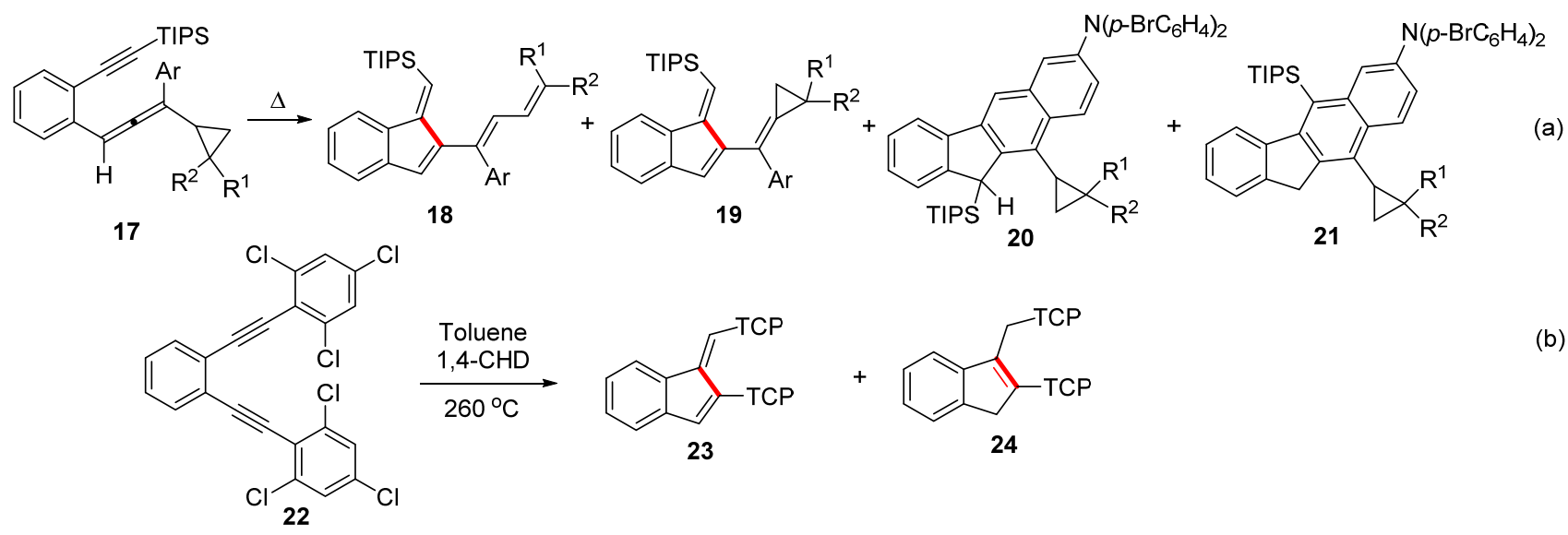

图式 4 热引发联烯一烯炔环化构建苯并富烯

Scheme 4 Synthesis of benzofulvenes by photo/thermal cyclization of enyne-allenes 
尝试反应. 由于会有副产物 24 的生成, 导致产率较低, 分离收率仅为 $19 \%$. 这种方法需要 $260{ }^{\circ} \mathrm{C}$ 的高温, 不能 广范应用于苯并富烯底物的合成. 该研究也没有对底物 进行更广泛的考察，但是证明了苯并烯二炔类的底物可 以通过热引发的方式, 发生 $C^{1}-C^{5}$ 的环化反应构建苯并 富烯.

2008 年, Schmittel 课题组 ${ }^{[20]}$ 报道了一例光催化烯 炔-联烯的 25 的 $\mathrm{C}^{2}-\mathrm{C}^{6}$ 环化反应. 根据经验他们合成了 联烯基末端羰基取代，炔基末端 TIPS 取代的底物 25, 通过篎选不同溶剂与照射波长, 由苯并烯炔-联烯 $\mathbf{2 5}$ 在 波长为 $300 \mathrm{~nm}$ 的光源照射下得到苯并富烯的三种产物, 这例反应的底物类型对该反应的影响比较大, 特定的几 个底物能得到苯并富烯的产物，最高分离收率可达 $51 \%$ (Scheme 5). Schmittel 课题组发展的这例光催化构建苯 并富烯的方法，可以以简单的方式获得了苯并富烯.
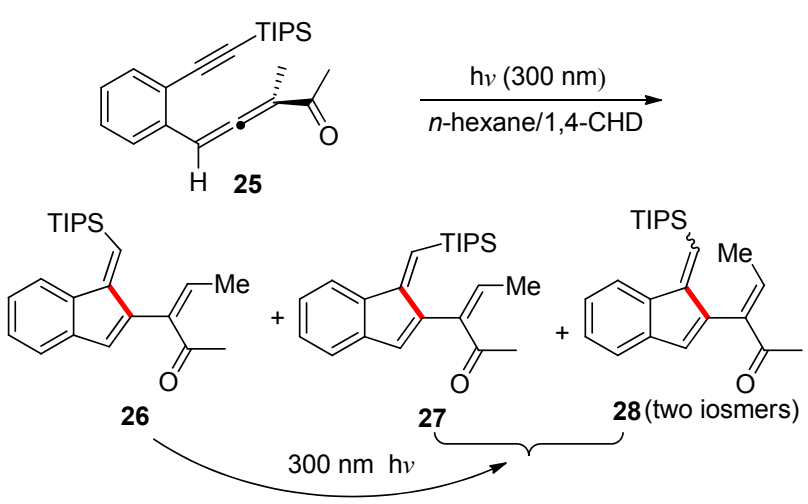

图式 5 光引发联烯一烯炔环化构建苯并富烯

Scheme 5 Synthesis of benzofulvenes by photo cyclization of enyne-allenes

光或者热引发烯二炔, 烯炔-联烯构建苯并富烯的 方法简单方便，一般来说联烯一烯炔类底物的环化反应 条件比二炔类底物的相对温和一些. 不过这些方法都不 可避免地会产生 Bergman 环化产物 $\left(\mathrm{C}^{1}-\mathrm{C}^{6}\right)$ 或 MyersSaito 环化产物 $\left(C^{2}-C^{7}\right)$ 等副产物. 此外, 已报道的相关方 法在底物的适用性以及环外双键构建等方面还有待进 一步的研究.

\section{2 过渡金属催化的串联环化反应}

近年来, 过渡金属在有机化学中的应用获得空前发 展, 使得高效地构建碳-碳以及碳一杂原子键日趋成熟. 这一发展也为苯并富烯骨架的构建提供了很多新途径. 在过渡金属催化下, 发生串联的环化反应为构建苯并富 烯类化合物提供了高效的方法.

碳氢键活化是近些年过渡金属催化的一个研究热 点. 应用 $\mathrm{Rh}, \mathrm{Ru}, \mathrm{Ir}, \mathrm{Pd}, \mathrm{Co}$ 等催化的 $\mathrm{C}-\mathrm{H}$ 键活化引发
反应，进而通过炔基插入和环化是构建苯并富烯的有效 方法.

2008 年, Shibata 等 ${ }^{[21]}$ 报道了一例羰基导向的铱催 化 $\mathrm{C}-\mathrm{H}$ 活化反应, 发展了以苯乙酮 $\mathbf{3 0}$ 和二苯基乙炔 29 为底物, $\left[\operatorname{Ir}(\operatorname{cod})_{2}\right] \mathrm{BF}_{4}(5 \mathrm{~mol} \%)$ 为催化剂, 邻位 $\mathrm{C}-\mathrm{H}$ 键活化构建邻烯基苯乙酮片段. 当铱催化体系改变为 $\left[\operatorname{Ir}(\operatorname{cod})_{2}\right]$ OTf (5 mol\%), 得到环化的主产物苯并富烯和 狮醇 ${ }^{[22]}$, 拓展底物后以中等至优秀的收率得到了一系 列环化的苯并富烯产物(Eq. 1). 但对于能够产生环外构 型的底物, 作者没有进行充分研究, 仅考察了一个底物, 尽管最终得到了单一构型的产物，但是没有确定构型. 该课题组又尝试了其它类型的铱络合物，不能得到环化 的苯并富烯产物, 而且这种方法需要以氯苯为溶剂并长 时间回流才能得到产物.
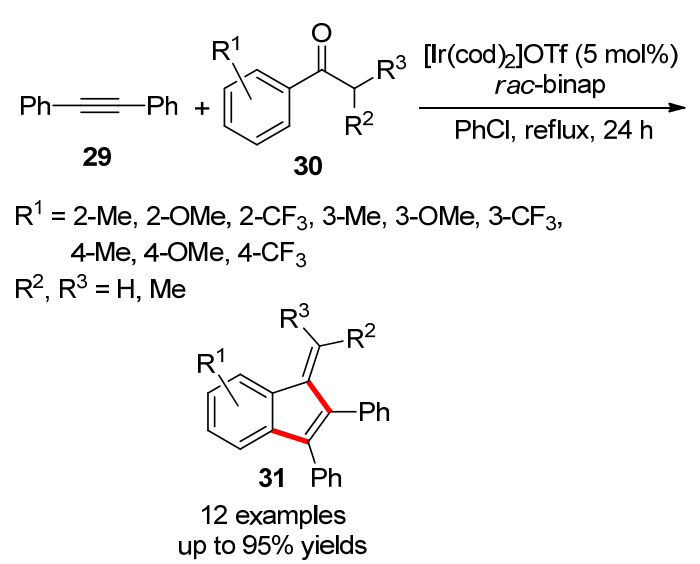

2011 年 Glorius 课题组 ${ }^{[23]}$ 报道了一例铑催化 $\mathrm{C}-\mathrm{H}$ 键活化构建苯并富烯衍生物的反应(Scheme 6). 在优化 条件下, 以 $\left[\mathrm{RhCp}^{*} \mathrm{Cl}_{2}\right]_{2}$ 和 $\mathrm{AgSbF}_{6}$ 为共同催化剂, 同时 有等物质的量的 $\mathrm{Cu}(\mathrm{OAc})_{2}$ 存在时，以高达 $99 \%$ 的分离 收率得到环化产物. 通过对反应条件的微调, 改变溶剂 和反应温度分别得到苯并富烯或者茆醇(non-dehydrative vs $\alpha$-route, $\beta$-route). 研究发现醋酸铜对这个反应很 重要. 作者根据实验现象推测, 认为铜在催化循环中通 过转金属化释放铑催化剂物种, 促进催化循环的进行. 作者还发现，底物的取代基不同会导致最终产物的不 同，也就是取代基会影响终产物. 在非脱水反应模式 (non-dehydrative)中，当 $\mathrm{R}^{1}$ 为异丙基，叔丁基等大位阻 的取代基, $\mathrm{R}^{2}$ 为拉电子基团时得到茚醇. $\mathrm{R}^{1}$ 为甲基或者 氢原子且 $\mathrm{R}^{2}$ 为推电子基团时则得到苯并富烯的产物 ( $\alpha$-route). Glorius 等发展的这一方法的官能团容忍性很 好, 无论是强的吸电子或者给电子取代基或者杂环化合 物的底物, 反应均可以进行, 还可以控制反应停留在狮 醇阶段. 但对于环外双键的构型，该方法无法有效的控 制. 

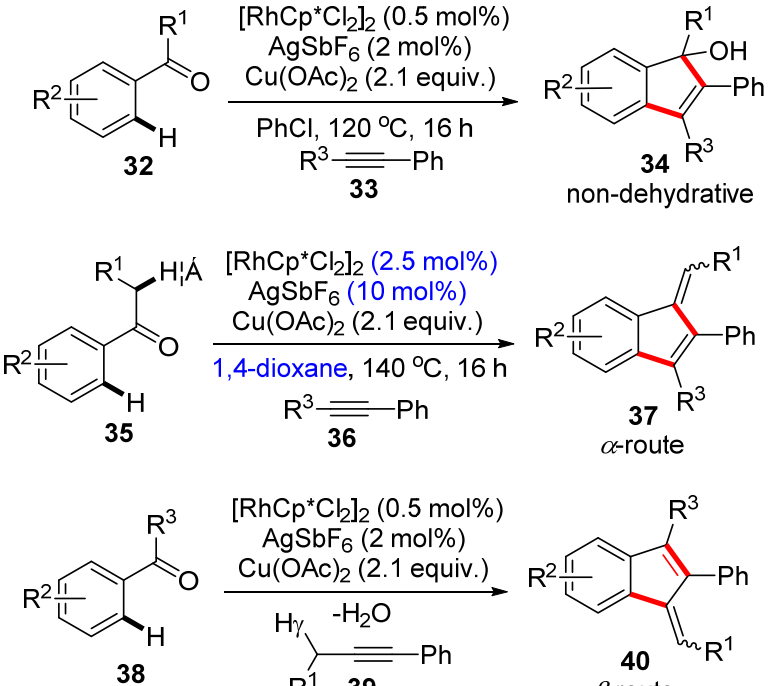

$\left[\mathrm{RhCp}{ }^{*} \mathrm{Cl}_{2}\right]_{2}(0.5 \mathrm{~mol} \%)$

$\mathrm{AgSbF}_{6}(2 \mathrm{~mol} \%)$ $\mathrm{Cu}(\mathrm{OAc})_{2}$ (2.1 equiv.)

38
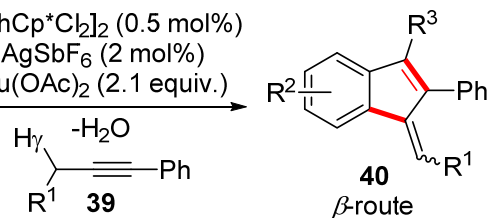

20 examples $40 \% \sim 99 \%$ yield

图式 6 铑催化的 $\mathrm{C}-\mathrm{H}$ 键活化构建苯并富烯

Scheme 6 Rhodium catalyzed synthesis of benzofulvenes

2012 年, 利用类似的反应底物, Jeganmohan 课题 组 ${ }^{[24 \mathrm{a}]}$ 报道了一类较廉价的钌催化的 $\mathrm{C}-\mathrm{H}$ 键活化构建 苯并富烯的方法. 反应与铑催化的 $\mathrm{C}-\mathrm{H}$ 活化类似, 利 用类似的底物 41 在 $\left[\mathrm{RuCl}_{2}(p\right.$-cymene $\left.)\right] 、 \mathrm{Cu}(\mathrm{OAc})_{2} \cdot \mathrm{H}_{2} \mathrm{O}$ (25 mol\%)作用下, 生成狮醇类产物 43 (Scheme 7). 其它 条件不变, 将 $\mathrm{AgSbF}_{6}$ 的用量增加至 $20 \mathrm{~mol} \%$, 得到苯并 富烯类产物 44. 但该反应在环外双键的构型方面并没 有取得进展, 这是苯乙酮类底物构建苯并富烯的缺点之<smiles>[R]C#C[R]C#N</smiles>

$43 \mathrm{R}^{1}$
$\left[\mathrm{RuCl}_{2}(p \text {-cymene })\right]_{2}(2 \mathrm{~mol} \%)$ $\mathrm{AgSbF}_{6}, \mathrm{Cu}(\mathrm{OAc})_{2} \cdot \mathrm{H}_{2} \mathrm{O}(25 \mathrm{~mol} \%)$ 21 examples $73 \% \sim 93 \%$ yields or<smiles>[R]/C=C1\C([R])=C([R])c2ccccc21</smiles>

(a)

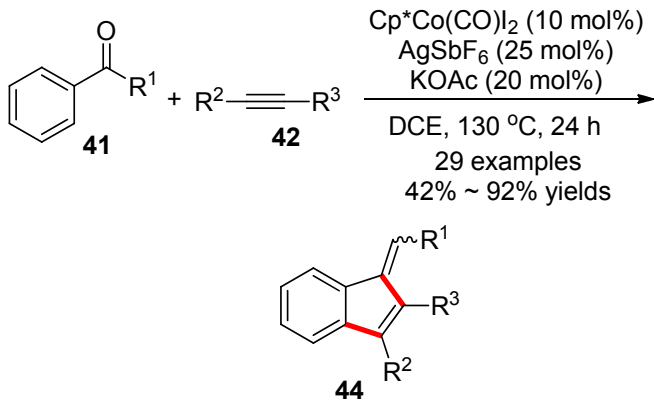

图式 7 钉催化的 $\mathrm{C}-\mathrm{H}$ 键活化构建苯并富烯

Scheme 7 Synthesis of benzofulvenes by ruthenium catalyzed $\mathrm{C}-\mathrm{H}$ bond activation
一，另一个缺点是反应会不可避免地产生副产物狮醇. 作者推测其反应机理与铑催化的 $\mathrm{C}-\mathrm{H}$ 键活化构建苯并 富烯的机理类似. 2019 年谭泽和朱钢国课题组使用钴催 化实现了同样的反应 ${ }^{[24 b]}$.

2015 年，华瑞茂课题组 ${ }^{[25]}$ 用新的底物二苯基乙炔 45 发展了一类钯催化的 $\mathrm{C}-\mathrm{H}$ 键活化构建苯富烯 46 的 方法(Scheme 8). 新底物的使用有效地避免了狮醇类副 产物的生成. 最终，二苯基乙炔在醋酸钯的催化下，在 $N, N$-二甲基乙酰胺中加入溴化锂、特戊酸铯等添加剂, 以中等偏上的收率得到了一系列苯基取代苯并富烯化 合物。<smiles>[R]c1ccc(C#Cc2ccc([As])cc2)cc1</smiles>
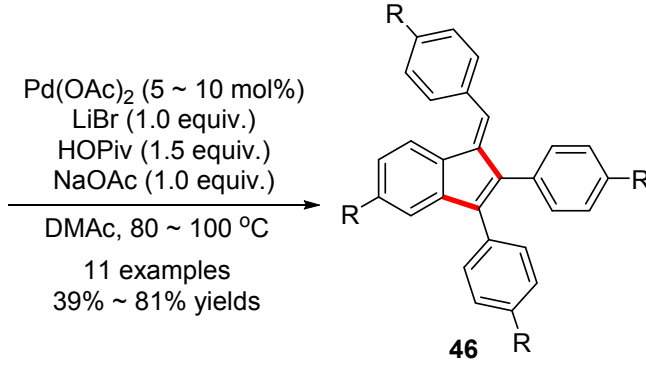<smiles>C(=C1C(=C/c2ccccc2)/Cc2ccccc2/1)\c1ccccc1</smiles>

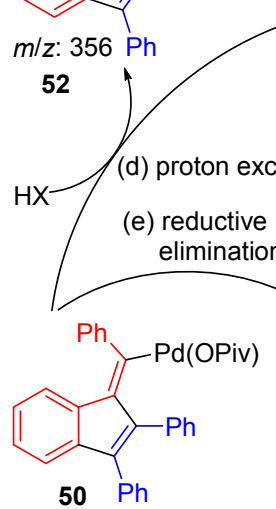

(c) intramolecular inserion

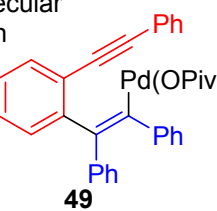
b) intermolecular

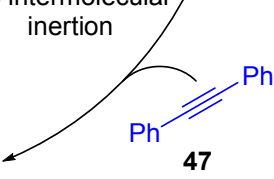

图式 8 钯催化的 $\mathrm{C}-\mathrm{H}$ 键活化构建苯并富烯

Scheme 8 Palladium catalyzed synthesis of benzofulvenes via $\mathrm{C}-\mathrm{H}$ activation

2019 年, 莊士卿课题组 ${ }^{[26]}$ 报道了一例类似的反应, 也是由两分子二芳基乙炔构建出苯并富烯骨架(Eq. 2).

除了 $\mathrm{C}-\mathrm{H}$ 活化引发的串联环化反应，过渡金属催 化的多种反应类型都可以引发相应的反应. 
<smiles>[R]c1ccc(C#[R])cc1</smiles>

53

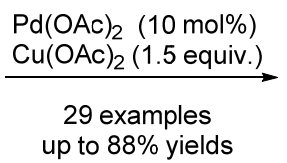

up to $88 \%$ yields

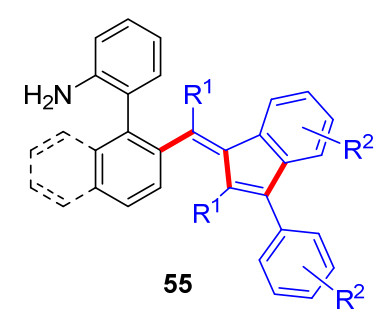

(2)

1996 年, Dyker 等 ${ }^{[27]}$ 报道了一例以卤代 1,1-二苯基2-溴代乙烯 $(\mathbf{5 6})$ 为底物, 多米诺的 Heck 反应构建苯并富 烯的方法(Scheme 9), 该反应先经历分子间 Heck 反应, 再发生分子内的 Heck 反应构建苯并富烯. 作者没有对 底物的官能团进行考察, 只从构建大环的角度对底物的 进行了拓展合成了一系列苯并富烯并环的结构.<smiles>[X]C=C(c1ccccc1)c1ccccc1</smiles>

56
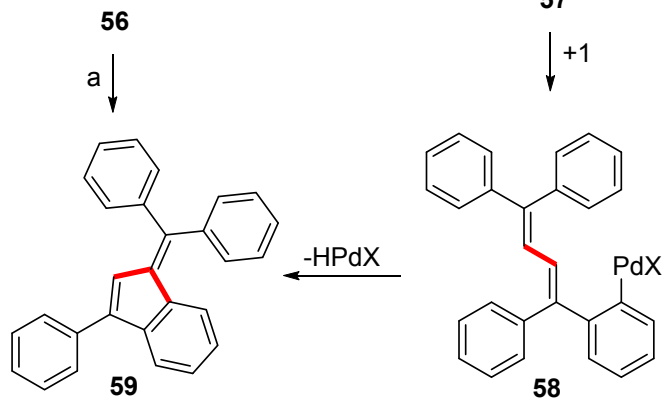

图式 9 钯催化多米诺 Heck 反应

Scheme 9 Palladium catalyzed domino heck reaction

2004 年, Singer 课题组 ${ }^{[28]}$ 开发了用 2-卤代苯乙腈 60 与乙氧基代丙烯酸乙酯(61)反应构建氰基取代苯并富烯 衍生物 62 的方法(Eq. 3). 作者合成苯并富烯仅作为合成 其他化合物的一个关键中间体, 并没有对底物的官能团 兼容性进行深入考察, 仅考察了几个简单的底物. 这种 方法合成了氭基取代的苯并富烯衍生物, 有利于苯并富 烯骨架的官能团化, 而且氯代的底物也同样适用于该反 应.

2006 年, Kan 课题组 ${ }^{[29]}$ 报道了一例钯催化的多步 Heck 反应, 由累积三烯构建苯并富烯的方法(Scheme 10). 尽管在 63 中有共轭醛基的存在, 但芳基钯物种仍 会高选择性地插入到 $C^{3}-C^{4}$ 之间, 形成六元环钯物种 64, 最后还原消除得到苯并富烯的衍生物 65 .

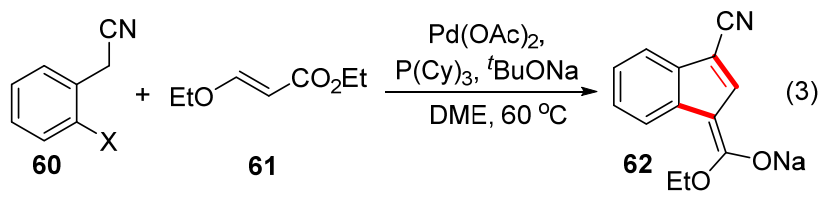<smiles>CCOC(O[Na])=C1C=C(C#N)c2ccccc21</smiles><smiles>COC(OC)=C1C=C(C#N)c2cc(OC)c(OC)cc21</smiles><smiles>CCOC(O[Na])=C1C=C(C#N)c2c(F)cccc21</smiles><smiles>CCOC(=O)C1=CC(C#N)c2ccc(C(F)(F)F)cc21</smiles>
$67 \%$<smiles>CCOC(O[Na])=C1C=C(C#N)c2ccc(F)cc21</smiles>

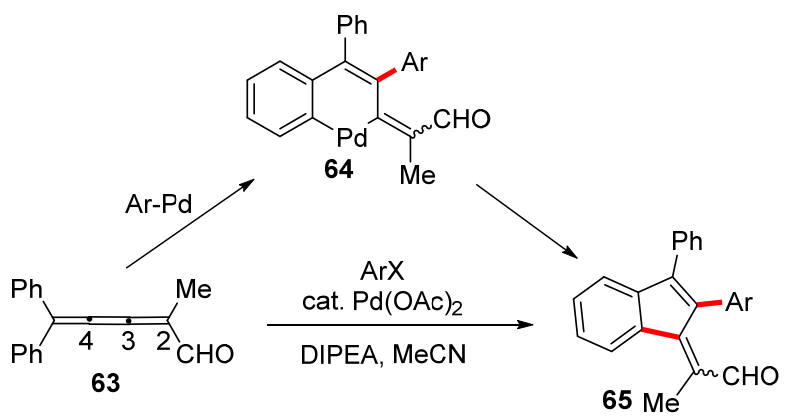<smiles>[R]c1ccc(C2=C(c3ccccc3)c3ccccc3C2=C(C)C=O)cc1</smiles>

$\mathrm{R}=\mathrm{H} \quad 59 \%, \mathrm{Z} / \mathrm{E}=3.6 / 1.0$

$\mathrm{R}=\mathrm{OMe} 46 \%, Z / E=3.6 / 1.0$

$\mathrm{R}=\mathrm{OMs} 39 \%, Z / E=2.4 / 1.0$

$\mathrm{R}=\mathrm{NO}_{2} \quad 59 \%, Z / E=4.3 / 1.0$

$\mathrm{R}=\mathrm{Br} \quad 59 \%, Z / E=2.7 / 1.0$

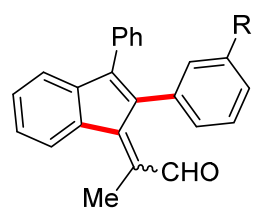

$\mathrm{R}=\mathrm{OMe} 47 \%, Z / E=2.3 / 1.0$ $\mathrm{R}=\mathrm{OMs} 62 \%, \mathrm{ZIE}=2.3 / 1.0$ $\mathrm{R}=\mathrm{NO}_{2} \quad 47 \%, \mathrm{Z} / \mathrm{E}=2.5 / 1.0$

图式 10 不对称累积烯烃多米诺反应构建苯并富烯 Scheme 10 Synthesis of benzofulvenes via domino reaction with unsymmetrically substituted cumulene

2006 年, Tobe 课题组 ${ }^{[30]}$ 合成了 1,6-二取代炔二烯结 构的底物 66, 在钯的催化下构建出桥联苯并富烯衍生 物 67 (Eq. 4). 这种刚性的大共轭桥联苯并富烯骨架的 分子, 是一类具有优异二阶非线性光学发色团(Second Order Nonlinear Optical Chromophores)的化合物, 可以 作为一些先进光学器件的核心原料.

2009 年，王剑波课题组 ${ }^{[31]}$ 报道了一例金催化的不 饱和三元环扩环反应构建苯并富烯的方法(Scheme 11). 不饱和底物 68 的碳碳双键与金离子进行配位, 然后三 元环开环形成金卡宾 71, 接着发生卡宾插入反应得到 72, 再在碱的作用下消除一分子乙酸得到产物 69. 该方 法研究的是简单的苯并富烯有衍生的合成, 并不涉及环 外双键的考察. 


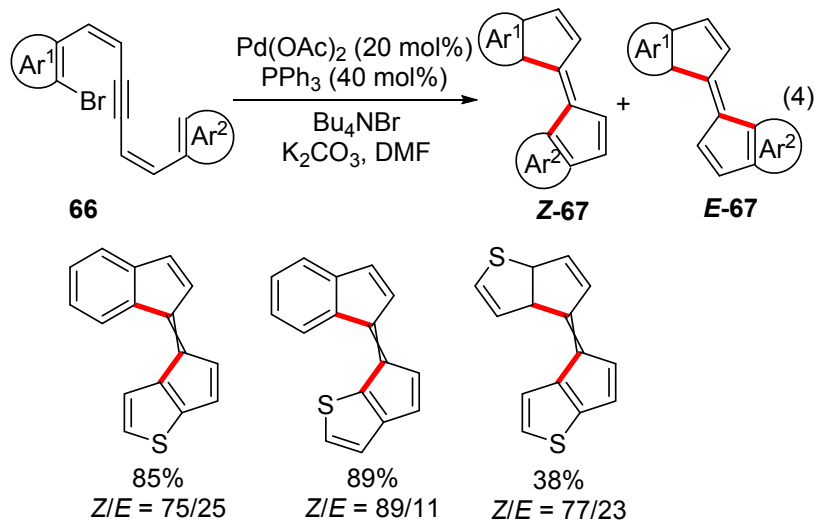

2010 年，该课题组随后又报道了两例偕二澳取代 烯基苯合成苯并富烯的方法. 分别使用 $\mathbf{7 5}^{[33 a]}$ 和 $7^{[33 b]}$ 为底物. 在醋酸钯的催化下, 以三苯基膦为配体, 加入 碱, 室温下发生分子内的 Heck 反应，接着与芳基嗍酸 发生 suzuki 反应构建苯并富烯衍生物(Scheme 12a). 这 种方法反应条件温和，收率等达到中等偏上，而且还避 免了环外双键产生混合物的情况.<smiles>CC(C)(C)C=Cc1ccccc1C=C(Br)C#Cc1ccccc1C=C(Br)Br</smiles>

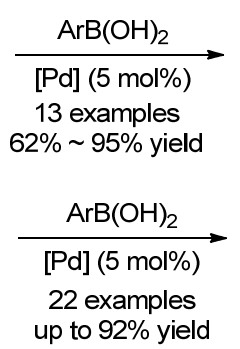

图式 12 钯催化 Suzuki/Heck 串联反应构建苯并富烯 Scheme 12 Pd-catalyzed tandem Suzuki/Heck reaction of 1(2,2-dibromovinyl)-2-alkenylbenzenes

同年, Lautens 课题组 ${ }^{[34]}$ 利用类似的溴代二烯基的 底物 77 以 $\mathrm{Pd}_{2} \mathrm{dba}_{3}$ 为钯源, 碳酸铯为碱, 三呋喃基膦为 配体, 在 $60{ }^{\circ} \mathrm{C}$ 的二氧六环和水的混合溶剂中得到苯并 富烯的衍生物 78 (Scheme 12b).

2010 年, Kim 课题组 ${ }^{[35]}$ 以 Baylis-Hillman 加成产物 为原料经过一步反应得到产物 79, 然后通过 Barbier 反 应得到 80, 接着发生分子内 Heck 反应消除构建顺反异 构体的苯并富烯混合物 81 (Scheme 13).

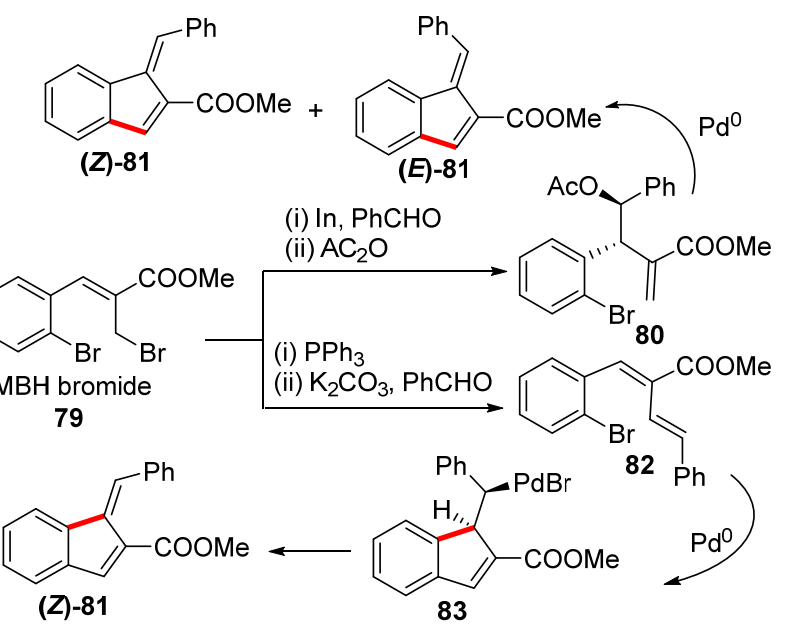

图式 13 由 Baylis-Hillman 加成产物构建苯并富烯 Scheme 13 Building benzofulvene derivatives from BaylisHillman adduct

2013 年, 该课题组 ${ }^{[36]}$ 改进了上述工作，采用反式共 轭二烯结构底物 82 , 通过控制碱的不同(三乙胺和碳酸 铯), 实现了对几个不同官能团底物环外双键的构型的 基本控制，分别得到主产物不同的 $\mathbf{8 1}$. 
2012 年, 张兴国课题组 ${ }^{[37]}$ 报道了一例钯催化的苯 并富烯衍生物的合成方法(Scheme 14). 该工作直接使 用氯代底物 84 为底物, 与钯发生氧化加成、炔烃插入接 着与硼酸发生 Suzuki-Miyaura 偶联反应，得到三氟甲基 化的苯并富烯. 该反应以串联的环化一偶联反应提供了 一个构建苯并富烯以及合成三氟甲基化苯并富烯的新 方法. 2018 年他们 ${ }^{[376]}$ 进一步地拓展了这一反应, 直接使 用有机胺为偶联片段合成吲哚或吡咯取代的苯并富烯.

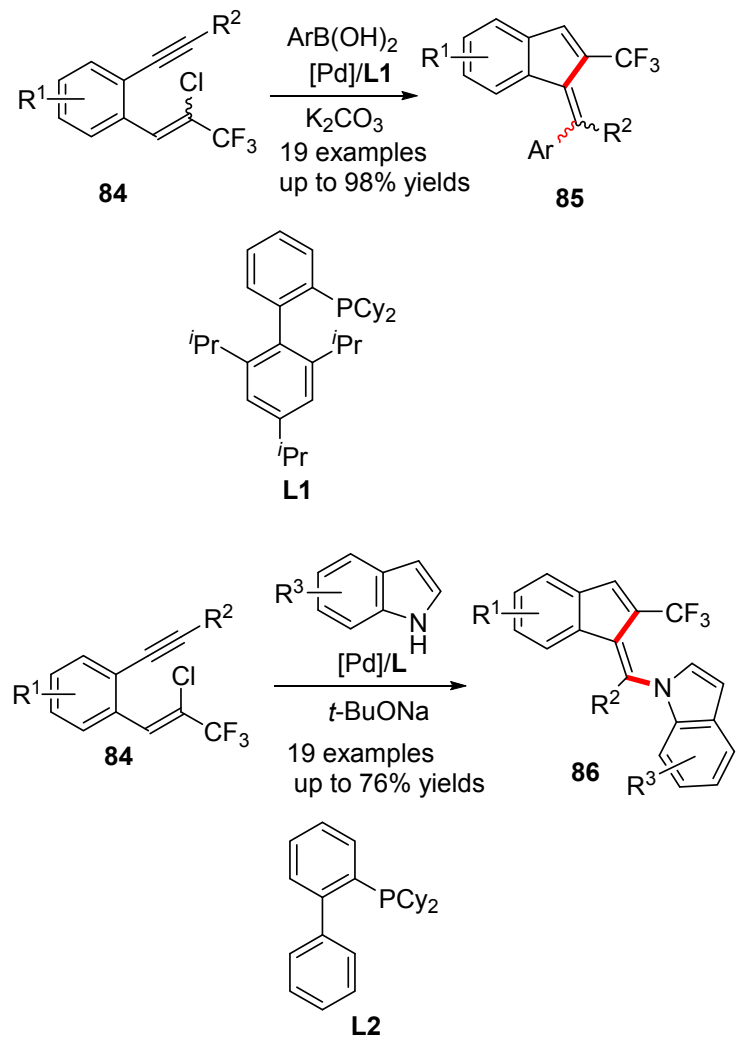

图式 14 钯催化的串联环化-偶联反应构建苯并富烯

Scheme 14 Synthesis of 2-CF - -benzofulvenes via Pd-catalyzed tandem carbocyclization-coupling reactions

2012 年, Sanz 课题组 ${ }^{[38 a]}$ 发展了一例金催化环化串 联反应构建苯并富烯衍生物的方法. 该方法也需要经历 金正离子物种，环化之后通过消除反应得到环外双键 (Eq. 6), 最终以较高的收率得到了 (E)-89 为主产物. 2015 年他们 ${ }^{[386]}$ 使用对甲苯磺酸催化实现了该反应的转化, 无需金属催化剂.

2014 年, 陈知远课题组 ${ }^{[39]}$ 发展了一例铂催化环化 串联反应构建苯并富烯衍生物的方法(Eq. 7). 作者认为 该反应首先经历了酰基 [3,3]-重排反应生成联烯中间体, 然后在金属铂的催化作用下关环，最后发生 $[1,5]$-酰基 迁移得到了环外双键为 $Z$ 构型的产物.

2013 年, 姚祝军课题组 ${ }^{[40]}$ 也报道了一例金催化的 苯并富烯衍生的合成方法 (Eq. 8). 他们设计了一类含羧

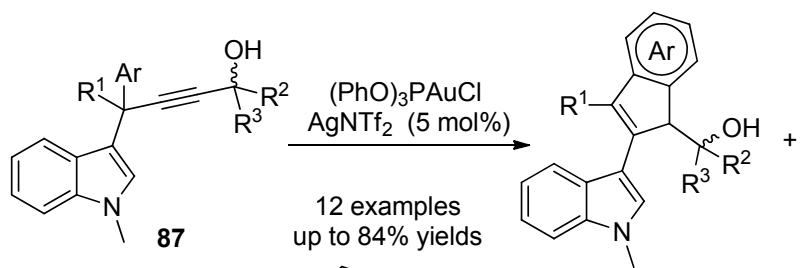<smiles>[R]C([R])=C1C(c2cn(C)c3ccccc23)=C([R])c2ccccc21</smiles>

88<smiles>[R]C#Cc1cc[R1]([H])cc1C(C#C[R])OC([R])=O</smiles>

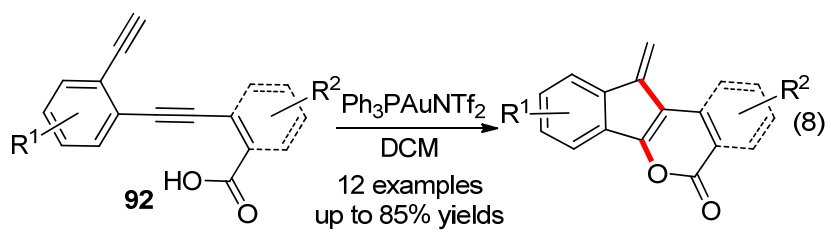

基的二炔基苯为底物, 以 $5 \mathrm{~mol} \% \mathrm{Ph}_{3} \mathrm{PAuNTf}_{2}$ 为催化剂, 在二氯甲烷溶液中, 得到了氧杂六元环并的苯并富烯衍 生物.

2015 年, Hamze 等 ${ }^{[41]}$ 报道了一例一锅法串联合成苯 并富烯的方法. 该方法以邻炔基腙类化合物 93 为底物, 通过配体的调控, 可以依次实现腙与卤代烃的偶联和成 环反应(Eq. 9).

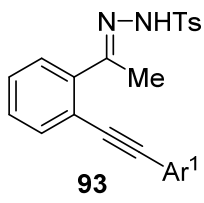

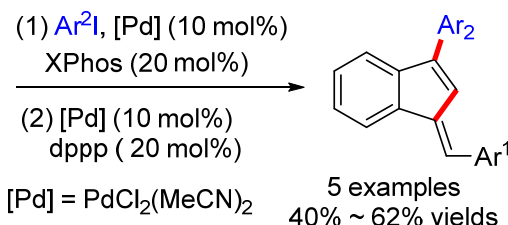

2016 年, Lee 课题组 ${ }^{[42]}$ 报道了铑催化的三氮唑脱氮 环化反应构建苯并富烯的方法(Scheme 15). 三氮唑 95 在二价铑存在的条件下脱除一分子的氮气，生成的铑一 卡宾中间体亲电进攻苯环，金属铑离去后重排得到苯并 富烯 96. 三氮唑类化合物可以由炔烃与叠氮通过铜催 化的 Click 反应得到. 94 和磺酰叠氮在催化剂 $\mathrm{CuTC}$ 和 $\mathrm{Rh}_{2}(S \text {-DOSP })_{4}$ 的催化下，可以一锅法合成苯并富烯. 这 一工作采用一系列设计新颖的底物, 最终得到了一系列 氨基官能团化的苯并富烯衍生物. 


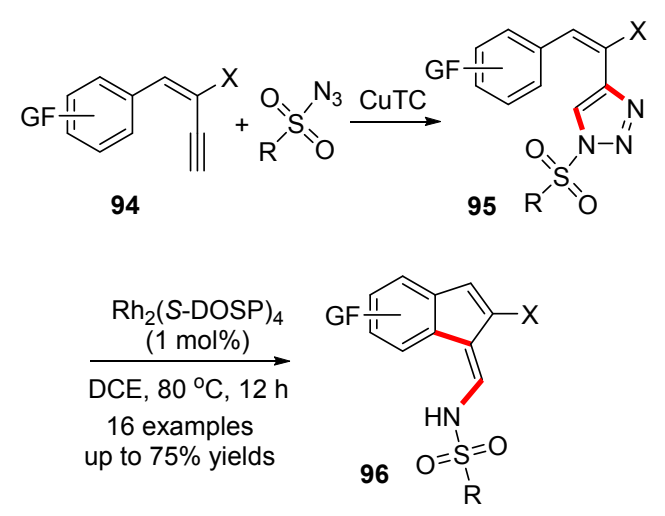

图式 15 一锅法合成胺基苯并富烯

Scheme 15 One-pot synthesis of amino substituted benzofulvenes

2018 年, 张扬会课题组 ${ }^{[43]}$ 报道了碘苯 97 、炔 98 和 二溴甲烷 99 在钯催化下发生三组分串联反应制备苯并 富烯的反应(Eq. 10). 作者认为该反应经历了环钯中间 体, 碘苯和炔烃与金属钯形成环钯物种后与二溴甲烷偶 联得到狮, 茚在碱性条件下与另一分子的二溴甲烷缩合 得到苯并富烯.

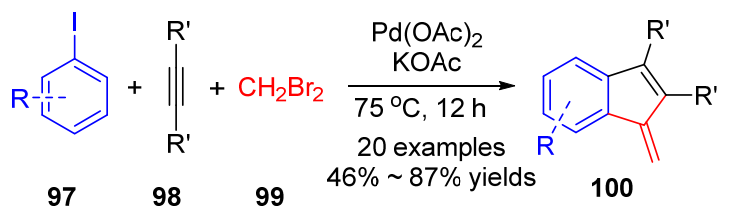

2019 年, 吴小锋课题 ${ }^{[44]}$ 组报道了一例钯催化的四 组分反应构建苯并富烯的反应(Eq. 11). 该方法使用简 单易得的原料，经过一步反应以中等收率得到多取代的 苯并富烯.

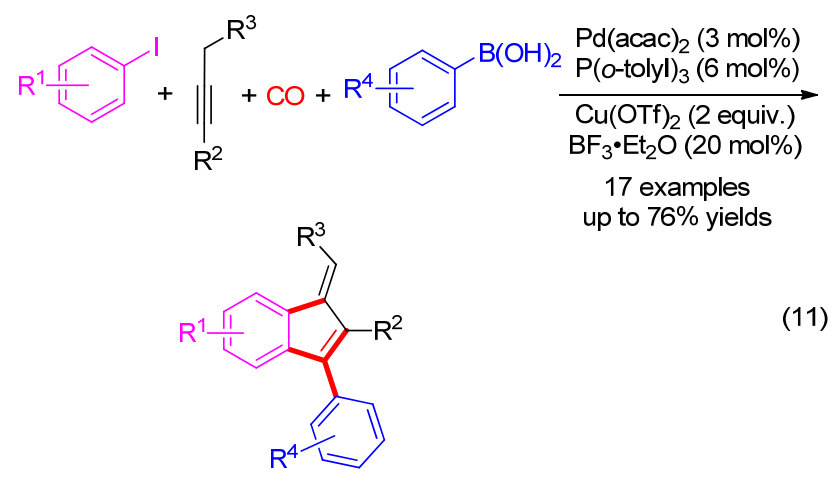

同年 Gogoi 等 ${ }^{[45]}$ 报道了一例钯催化的 C-H 活化/炔 烃插入的脱羰环化反应(Scheme 16). 利用此方法, 可以 很方便地合成含苯酞片段的苯并富烯. 该反应以羟基荎 醌 101 为底物, 与炔烃在乙酸钯催化下, 经过 C-H 活化, 炔烃插入, 重排脱羰, 还原消除得到产物 102. 钯在乙 酸铜的氧化下再生二价钯实现了催化循环.

Hashmi 课题组 ${ }^{[46]}$ 报道了双金催化的非共轭二炔底

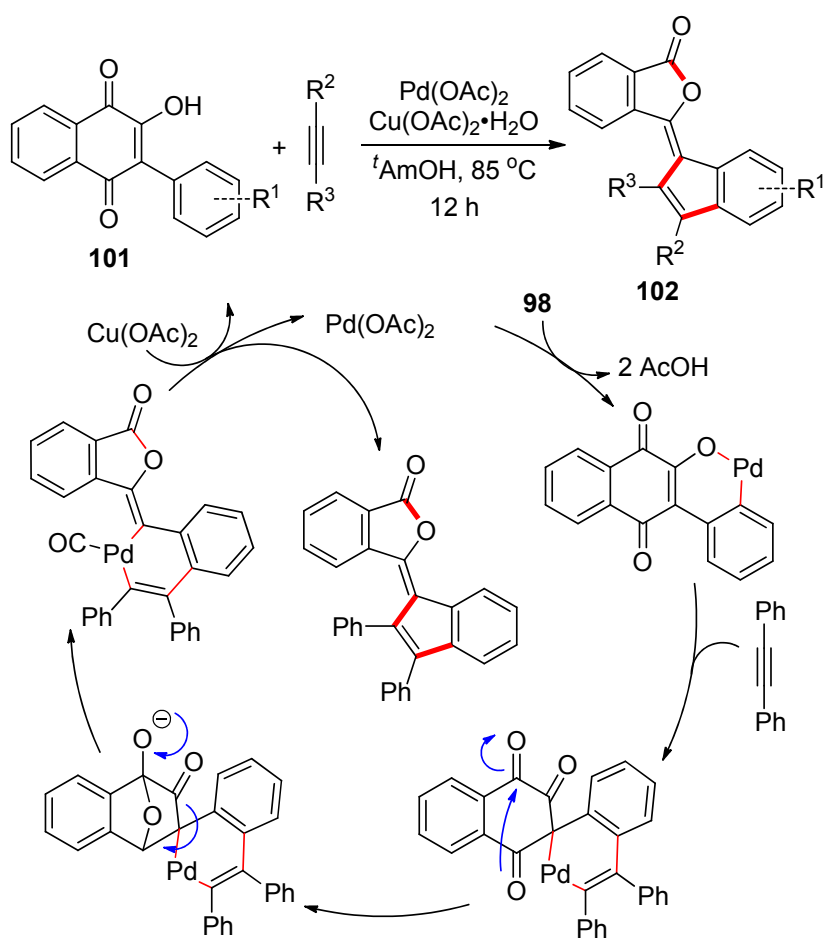

图式 16 钯催化合成含苯酞片段的苯并富烯

Scheme 16 Synthesis of phthalide-fused benzofulvenes

物 103 构建刚性结构的的苯并富烯衍生物的方法(Eq. 12). 该方法反应条件温和, 底物适用范围比较广泛.

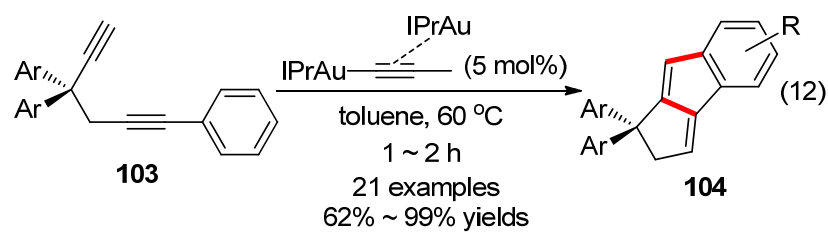

通过不同策略和合成砌块构建环戊二烯环是苯并 富烯的主要合成方法. 2019 年 Blond 等 ${ }^{[47]}$ 报道了一例完 全不同的策略，通过直接构建苯环来合成苯并富烯(Eq. 13). 使用预组织好的三炔化合物为底物在 $\left[\mathrm{Rh}(\mathrm{CO})_{2}\right]_{2}$ 的 催化下，炔烃芳构化构建苯环得到多环骨架的产物. 反 应还会生成环庚三烯酮骨架的副产物. 该方法可以合成 多取代的非对称的多环苯并富烯.

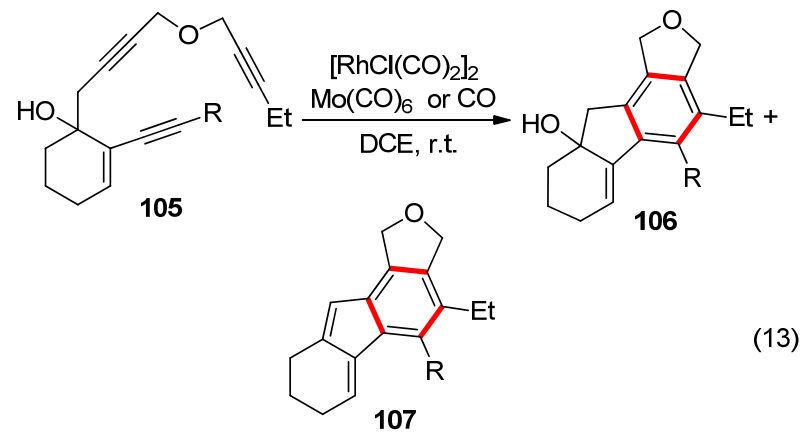




\section{3 亲电或亲核试剂进攻引发的环化反应}

利用亲电或者亲核试剂进攻引发环化反应的苯并 富烯合成研究很早就有报道. 1969 年 Reichardt 课题 组 ${ }^{[48]}$ 研究了简单亲电试剂, 如溴化氢、水、碘或溴等对 苯并烯二炔类底物 108 的影响(Scheme 17). 当加入溴化 氢气体或者溴化氢的乙酸溶液会产生两种异构体 106、

113, 加入溴或者碘单质则会生成二溴代或者二碘代的 苯并富烯衍生物 111, 而加入溴和甲醇得到的是溴代狮 的产物 114.

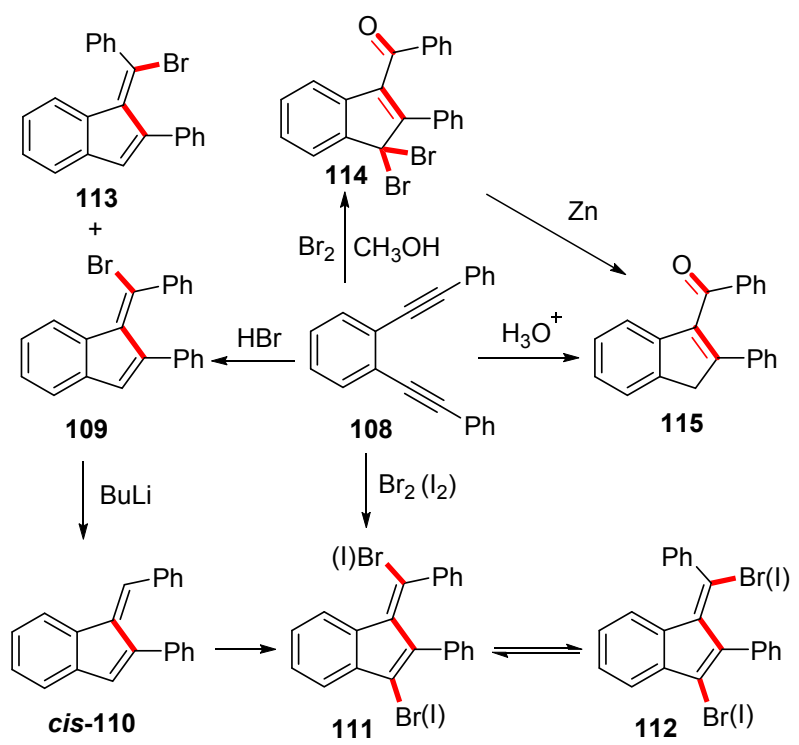

图式 17 引入亲电试剂促进环化反应构建苯并富烯

Scheme 17 Enediyne cyclization promoted by electrophiles to build benzofulvene derivatives

2003 年, Schreiner 课题组 ${ }^{[49 a]}$ 报道了一例构建多苯 取代苯并富烯的方法(Eq. 14). 该方法采用烯炔化合物 116, 与单质溴反应得到多芳基取代的溴代苯并富烯. 2017 年 Sanz 等 ${ }^{[49 b]}$ 利用相同的策略, 使用 NIS 为亲电试 剂, 得到了碘代苯并富烯.

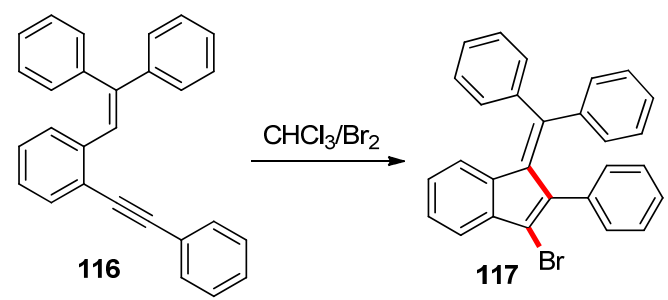

2007 年, 黄宪课题组 ${ }^{[50]}$ 报道了三氯化铝促进的酰 氯和亚烃基环丙烷开环/环化串联反应构建苯并富烯的 反应(Eq. 15). 该方法在温和的条件下可以方便地合成 官能团化的苯并富烯, 而且高选择性地生成了 Z-构型 的环外双键.

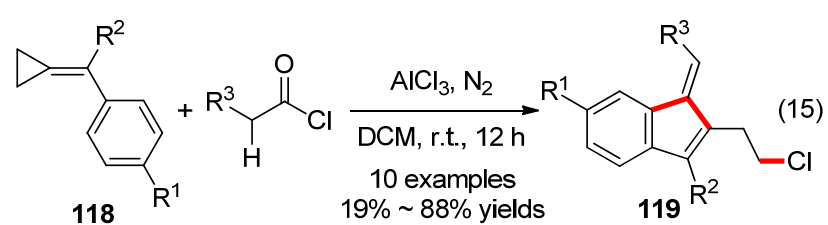

2014 年, 陈知远课题组 ${ }^{[51]}$ 报道了一例 $\mathrm{BiCl}_{3}$ 或 $\mathrm{TsOH}$ 促进的串联反应合成三氟甲硫基取代的苯并富烯 (Eq. 16). 使用 $N$-(三氟甲基硫代)苯胺提供亲电的三氟 甲基硫正离子，可以促进二炔的亲电关环，并有利于氯 离子或对甲苯磺酸离子的亲核反应.

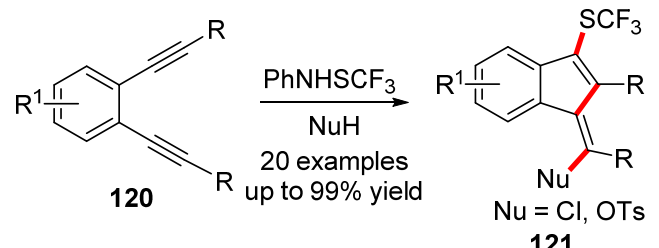

2014 年, Martinelli 等 ${ }^{[52]}$ 先通过两步简单的反应合 成 1,2-二芳基炔基苯，然后用碘促进环化构建碘代苯并 富烯，通过偶联反应，最终得到多取代苯并富烯的骨架 的共轭分子(Eq. 17).

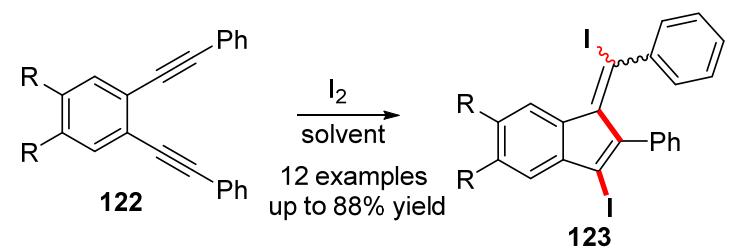

\section{4 自由基引发的环化反应}

2001 年, Schreiner 课题组 ${ }^{[53]}$ 用末端为羰基取代的烯 二炔为底物, 以 TEMPO 作为引发剂时, 在甲苯中回流 得到了 5-Exo-Dig 型关环中间体, 再算取氢后得到了富 烯产物. 但该方法用于苯并富烯衍生物的合成时，只是 发生了 5-Exo-Dig 型关环反应，得到 Bergman 环化产物， 没有苯并富烯生成.

2004 年, Alabugin 课题组 ${ }^{[54]}$ 尝试用自由基环化的方 法合成苯并富烯. 他们使用传统的烯二炔类化合物为底 物，在 AIBN 引发下以三丁基锡氢作为自由基源，首次 合成了锡取代的苯并富烯衍生物，经过酸化得到中等产 率的苯基取代苯并富烯(Scheme 18). 这种方法不同于 Bergman 环化的双自由基机理，是引发剂引发的自由基 链式反应. 而且该方法不能有效控制环外的双键的构 型, 得到的是 $Z / E$ 构型的混合物.

随后, Alabugin 课题组 ${ }^{[55]}$ 对苯并富烯产物中环外烯 烃中氢原子的来源进行了研究(Scheme 19). 通过気代 实验，发现氢原子来源于叔丁基锡氢，证实了该反应是 


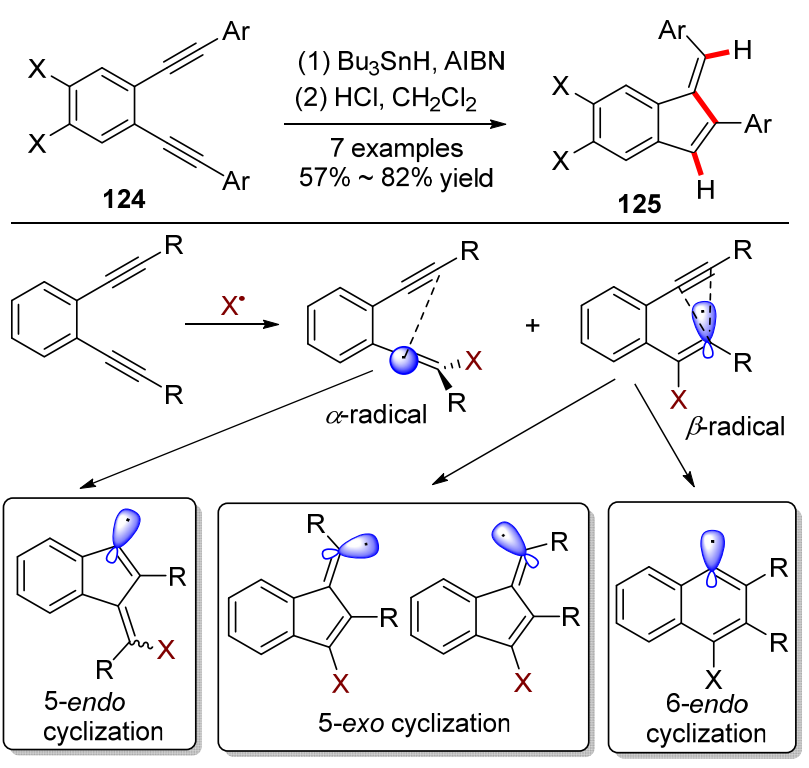

图式 18 自由基促进环化构建苯并富烯

Scheme 18 Radical induced synthesis of benzofulvenes

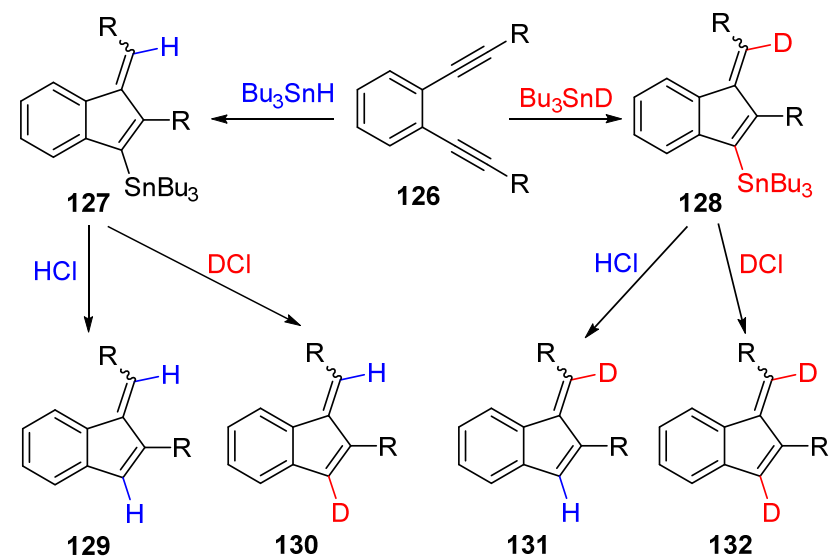

图式 19 氝代实验

Scheme 19 Deuterium-labeling experiments

自由基链式反应. 该研究为合成稳定同位素标记的苯并 富烯类化合物提供了一个有益的参考.

2019 年, 姜波等 ${ }^{[56]}$ 报道了一例自由基引发的构建 苯并富烯的反应(Eq. 18). 他们使用 $\mathrm{DABCO} \cdot\left(\mathrm{SO}_{2}\right)_{2}$ 复合 物作为引发剂, 与重氮盐 133 发生单电子转移, 二氧化 硫插入生成磺酰基自由基并释放出氮气, 磺酰基自由基 进攻另一分子炔烃 134, 进而发生串联的自由基环化反 应生成具有苯并富烯骨架的多环砜类化合物 $\mathbf{1 3 5}$.

\section{5 酸或碱促进的环化反应}

2009 年, Gandon 课题组 ${ }^{[57]}$ 报道了酸催化合成苯并 富烯的方法(Eq. 19). 该方法使用一类含有 $\alpha$-差基联烯 骨架的底物 136, 在酸的作用下发生 Nazarov-Type 的环 化构建苯并富烯. 研究发现, 路易斯酸和布朗斯特酸, 如 $\mathrm{ZnCl}_{2}, \mathrm{Zn}(\mathrm{OTf})_{2}, \mathrm{Cu}(\mathrm{OTf})_{2}, \mathrm{AgOTf}, \mathrm{TfOH}, \mathrm{H}_{3} \mathrm{PMo}_{12^{-}}$
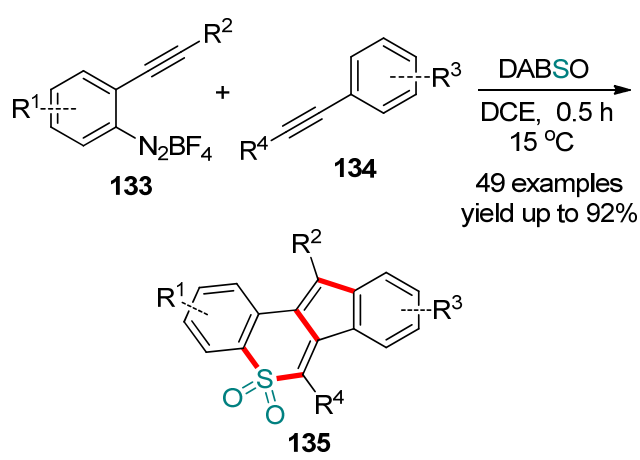

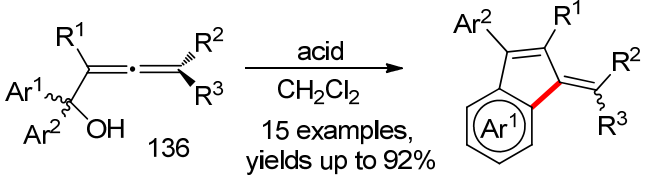

$\mathrm{O}_{40}$ 等, 都可以催化该反应.

2014 年, Coelho 课题组 ${ }^{[58]}$ 报道了一例 $p$ - TsOH 催化 的环化反应构建苯并富烯的方法(Scheme 20). 该方法 以四芳基-2-丁炔-1,4-二醇 137 为底物, 在催化量的 $p$ $\mathrm{TsOH}$ 作用下，与慕酚反应快速生成多取代的苯并富烯 138. 底物 137 可以由二苯基甲酮与乙炔基锂反应得到, 然后在酸的催化下与䒺酚发生傅克烷基化反应后异构 化为联烯的中间体，然后进行环化反应得到最终的苯

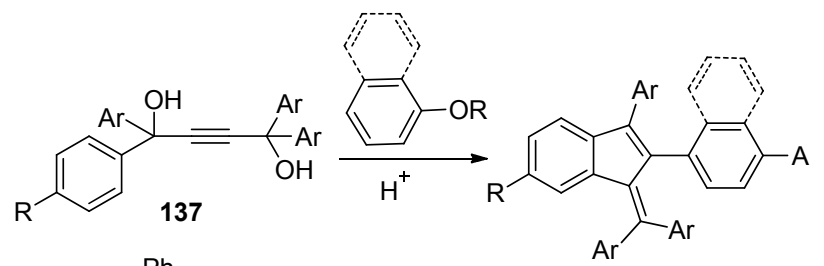

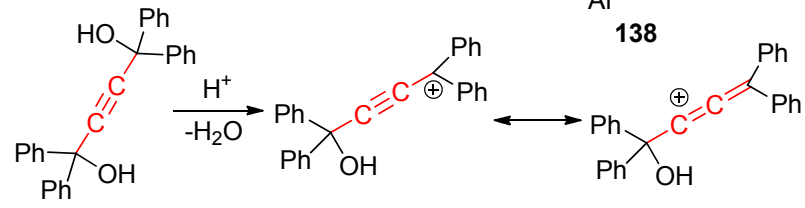<smiles>C[PH3+][CH-]C(=C=C(c1ccc(O)c2ccccc12)C(O)(c1ccccc1)c1ccccc1)c1ccccc1</smiles><smiles></smiles>

图式 20 酸催化构建多芳基取代苯并富烯衍生物

Scheme 20 Acid catalyzed synthesis of multi-substituted benzofulvenes 
并富烯产物. 这个方法特点是底物易于制备, 反应简单, 能得到高度取代的多芳基苯并富烯.

2014 年, Matsubara 课题组 ${ }^{[59]}$ 发展了锂催化的 Nazarov-Type 环化构建苯并富烯的合成方法(Eq. 20), 相比过渡金属催化的反应，锂催化对环境更加友好.
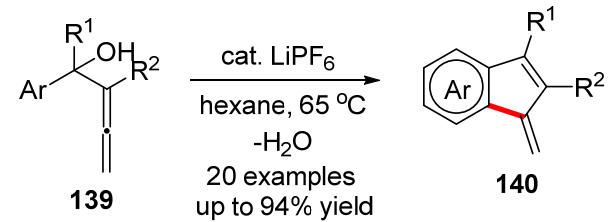

2019 年 Ingleson 等 ${ }^{[60]}$ 报道了二炔基苯硼化环化合 成苯并富烯的硼酸衍生物(Scheme 21). 该反应以路易 斯酸 $\mathrm{BCl}_{3}$ 引发炔烃环化, 同时又作为硼源. 当两性离子 生成后, 一个反应途径是㩲取一个 $\mathrm{Cl}$ 离子生成氯代苯 并富烯硼酸, 另一反应途径是进攻苯环, 生成二苯并戊 搭烯. 使用带正电荷的 $\mathrm{Cl}_{2} \mathrm{~B}$ (lutidine) ${ }^{+}$时, 反应更倾向于 生成二苯并戊搭烯 143 , 而加入 $\left(\mathrm{NBu}_{4}\right) \mathrm{BCl}_{4}$ 后, 则可以 增加苯并富烯 142 的比例.

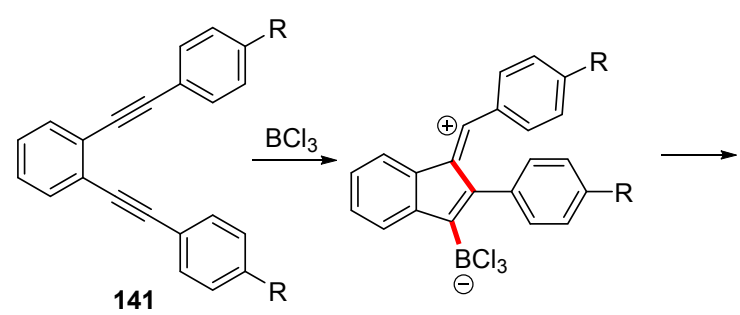

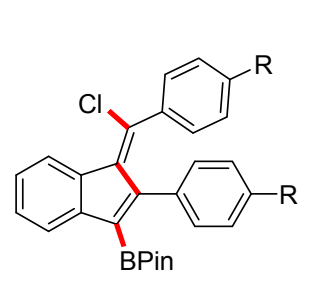

142

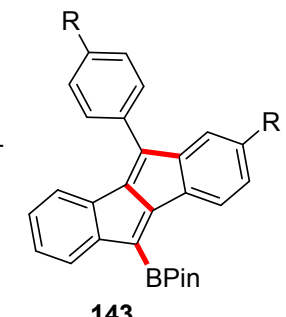

143
图式 21 合成含嗍苯并富烯

Scheme 21 Synthesis of boron substituted benzofulvenes

\section{6 总结与展望}

\section{1 总结}

由于苯并富烯在不同领域的广泛用途, 吸引了众多 有机化学家进行相关的合成研究. 一直以来, 传统的碱 催化苯并环戍二烯与醛酮缩合的方法存在底物的官能 团兼容性比较差的问题. 后来报道的以烯二炔和烯炔联烯类化合物为底物合成苯并富烯的方法丰富了苯并 富烯的合成途径. 但早期, 这种底物合成难度比较大, 随着金属催化的偶联反应的发展, 这种底物的合成难度
减小，使这种方法又有了新的活力. 利用烯二炔和烯 炔一联烯类底物, 早期主要是引入亲电试剂来加速环化 反应的进行，后来逐渐发展了光催化或热引发的环化反 应. 但是利用这类底物进行环化反应，不可避免的会产 生 $\mathrm{C}^{1}-\mathrm{C}^{6}$ 环化的苯环产物, 因为这类环化需要的能量较 低.

21 世纪之后, 随着各种偶联反应(Heck, Suzuki)和 $\mathrm{C}$ - H 键活化应用于苯并富烯骨架的合成, 用于苯并富 烯合成的底物变得丰富多样. 苯以酮类的底物可以通过 过渡金属催化的 $\mathrm{C}-\mathrm{H}$ 键活化与乙炔类的底物进行偶联 构建苯并富烯，不过这类方法总是会伴有狮醇类副产物 生成. 偕二溴烯炔类的底物通过分子内的偶联反应直接 构建苯并富烯产物，这类方法反应条件温和，改善了以 往苯并富烯骨架合成需要剧烈条件的状况, 然而底物实 用性范围还有待进一步的研究.

近期合成苯并富烯骨架的方法更加丰富, 通过设计 合适串联反应，可以以简单的底物，一锅合成结构复杂 的苯并富烯产物. 底物方面，既有多联烯的底物应用于 苯并富烯的合成，又有累积烯烃类型的底物来合成苯并 富烯骨架，饱和三元环也可以通过金催化构建苯并富 烯.

\section{2 展望}

虽然苯并富烯的合成已发展出了许多的方法，但仍 有问题需要就解决. 例如环外双键的构型控制问题就一 直没有很好的解决办法. 虽然对于一些特殊底物可以实 现有效控制，但是不具有普适性. 发展一类可以控制苯 并富烯环外双键构型，而且对广泛底物适用的方法很有 必要. 另外合成苯并富烯的底物基本都含有碳碳参键或 者联烯, 对于仅含有碳碳双键的底物研究够少, 未来这 方面将有望成为苯并富烯合成的趋势.

\section{References}

[1] Thiele, J. Chem. Ber. 1900, 33, 666.

[2] Yates, P. V. Adv. Alicyclic Chem. 1968, 2, 59

[3] Simple examples for application, see: (a) Kosaka, Y.; Kitazawa, K.; Inomata, S.; Ishizone, T. ACS Macro Lett. 2013, 2, 164. (b) Cappelli, A.; Galeazzi, S.; Giuliani, G.; Anzini, M.; Grassi, M.; Lapasin, R.; Grassi, G.; Farra, R.; Dapas, B.; Aggravi, M.; Donati, A.; Zetta, L.; Boccia, A. C.; Bertini, F.; Samperi, F.; Vomero, S. Macromolecules 2009, 42, 2368.

[4] (a) Douglas, A. W.; Larsen, R. D.; Verhoeven, T. R.; Reider, P. J. Tetrahedron Lett. 1995, 36, 3993.

(b) Shanmugam P.; Rajasingh, P. Chem. Lett. 2005, 34, 1494.

[5] (a) Walters, M. J.; Blobaum, A. L.; Kingsley, P. J.; Felts, A. S.; Sulikowski, G. A.; Marnett, L. J. Bioorg. Med. Chem. Lett. 2009, 19, 3271 .

(b) Felts, A. S.; Siegel, B. S.; Young, S. M.; Moth, C. W.; Lybrand, T. P.; Dannenberg, A. J.; Marnett, L. J.; Subbaramaiah, K. J. Med. Chem. 2008, 51, 4911 .

(c) Korte, A.; Legros, J.; Bolm, C. Synlett 2004, 2397.

(d) Maguire, A. R.; Papot, S.; Ford, A.; Touhey, S.; O'Connor, R.; Clynes, M. Synlett 2001, 41. 
[6] (a) Snyder, S. A.; Zografos, A. L.; Lin, Y. Angew. Chem., Int. Ed. 2007, 46, 8186.

(b) Jeffrey, J. L.; Sarpong, R. Tetrahedron Lett. 2009, 50, 1969.

(c) Singer, R. A.; McKinley, J. D.; Barbe, G.; Farlow, R. A. Org. Lett. 2004, 6, 2357.

[7] (a) Cappelli, A.; Galeazzi, S.; Giuliani, G.; Anzini, M.; Donati, A.; Zetta, L.; Mendichi, R.; Aggravi, M.; Giorgi, G.; Paccagnini, E.; Vomero, S. Macromolecules 2007, 40, 3005.

(b) Cappelli, A.; Galeazzi, S.; Giuliani, G.; Anzini, M.; Grassi, M.; Lapasin, R.; Grassi, G.; Farra, R.; Dapas, B.; Aggravi, M.; Donati, A.; Zetta, L.; Boccia, A. C.; Bertini, F.; Samperi, F.; Vomero, S. Macromolecules 2009, 42, 2368.

(c) Cappelli, A.; Paolino, M.; Grisci, G.; Giuliani, G.; Donati, A.; Mendichi, R.; Boccia, A. C.; Botta, C.; Mroz, W.; Samperi, F.; Scamporrino, A.; Giorgi, G.; Vomero, S. J. Mater. Chem. 2012, 22, 9611.

(d) Kosaka, Y.; Kitazawa, K.; Inomata, S.; Ishizone, T. ACS Macro Lett. 2013, 2, 164.

(e) Cappelli, A.; Villafiorita-Monteleone, F.; Grisci, G.; Paolino, M.; Razzano, V.; Fabio, G.; Giuliani, G.; Donati, A.; Mendichi, R.; Boccia, A. C.; Pasini, M.; Botta, C. J. Mater. Chem. C 2014, 2, 7897.

(f) Cappelli, A.; Razzano, V.; Paolino, M.; Grisci, G.; Giuliani, G.; Donati, A.; Mendichi, R.; Samperi, F.; Battiato, S.; Boccia, A. C.; Mura, A.; Bongiovanni, G.; Mroz, W.; Botta, C. Polym. Chem. 2015, 6, 7377.

(g) Kosaka, Y.; Kawauchi, S.; Goseki, R.; Ishizone, T. Macromolecules 2015, 48, 4421.

(h) Wang, W.; Schlegel, R.; White, B. T.; Williams, K.; Voyloy, D.; Steren, C. A.; Goodwin, A.; Coughlin, E. B.; Gido, S.; Beiner, M.; Hong, K.; Kang, N.-G.; Mays, J. Macromolecules 2016, 49, 2646.

(i) Chen. S.-D.; Li, Q.-P.; Sun, S.-Y.; Ding, Y.; Hu, A.-H. Macromolecules 2017, 50, 534.

[8] (a) Fischer, M.; Oswald, T.; Ebert, H.; Schmidtmann, M.; Beckhaus, R. Organometallics 2018, 37, 415.

(b) Rogers, J. S.; Lachicotte, R. J.; Bazan, G. C. Organometallics 1999, 18, 3976.

[9] Habaue, S.; Sakamoto, H.; Okamoto, Y. Polym. J. 1997, 29, 384.

[10] Jaquith, J. B.; Guan, J.; Wang, S.; Collins, S. Organometallics 1995, 14, 1079.

[11] Yuki, K.; Susumu, K.; Raita, K.; Takashi, I. Macromolecules 2015, $48,4421$.

[12] Grissom, J. W.; Gunawardena, G. U.; Klingberg, D.; Huang, D. Tetrahedron 1996, 52, 6453.

[13] (a) Mers, A. G.; Kuo, E. Y.; Finney, N. S. J. Am. Chem. Soc. 1989, 111, 8057 .

(b) Nagata, R.; Yamanaka, H.; Okazaki, E.; Saito, I. Tetrahedron Lett. 1989, 30, 4995.

[14] Matthias, P.; Alexander, W.; Peter, R. S. J. Phys. Chem. A 2001, $105,9265$.

[15] Schmittel, M.; Strittmatter, M.; Kiau, S. Tetrahedron Lett. 1995, 36, 4975.

[16] Schmittel, M.; Kiau, S.; Siebert, T.; Strittmatter, M. Tetrahedron Lett. 1996, 37, 7691 .

(b) Schmittel, M.; Maywald, M.; Strittmatter, M. Synlett 1997, 165

[17] Igor, V. A.; Serguei, V. K. J. Am. Chem. Soc. 2002, 124, 9052

[18] Schmittel, M.; Mahajan, A. A.; Bucher, G.; Bats, J. W. J. Org. Chem. 2007, 72, 2166.

[19] Vavilala, C.; Byrne, N.; Kraml, C. M.; Douglas, M.; Pascal, J. R. J. Am. Chem. Soc. 2008, 130, 13549.

[20] Bucher, G.; Mahajan, A.; Schmittel, M. J. Org. Chem. 2008, 73, 8815.

[21] Tsuchikama, K.; Kasagawa, M.; Hashimoto, Y.; Endo, K.; Shibata, T. J. Organomet. Chem. 2008, 693, 3939.

[22] Tsuchikama, K.; Kasagawa, M.; Endo, K.; Shibata, T. Synlett 2010, $1,97$.

[23] Patureau, F, W.; Besset, T.; Kuhl, T.; Glorius, J. F. J. Am. Chem. Soc. 2011, 133, 2154.
[24] (a) Chinnagolla, R. K.; Jeganmohan, M. Eur. J. Org. Chem. 2012, 417.

(b) Yu, Y.; Wu, Q.; Liu, D.; Hu, L.; Yu, L.; Tan, Z.; Zhu, G. J. Org. Chem. 2019, 84, 7449 .

[25] Guo, B.; Zheng, L.Y.; Zheng, Z. L.; Hua, R. M. J. Org. Chem. 2015, 80, 8430 .

[26] Raju, S.; Hsiao, H.-C.; Thirupathi, S.; Chen, P.-L.; Chuang, S.-C. Adv. Synth. Catal. 2019, 361, 683.

[27] Dyker, G.; Nerenz, F.; Siemsen, P.; Bubenitschek, P.; Jones, P. G. Chem. Ber. 1996, 1265.

[28] Singer, R. A.; McKinley, J. D.; Barbe, G.; Farlow, R. A. Org. Lett. 2004, 6, 2357.

[29] Furuta, T.; Asakawa, T.; Iinuma, M.; Fujii, S.; Tanaka, K.; Kan, T. Chem. Commun. 2006, 3648.

[30] Abdur Rahman, S. M.; Sonoda, M.; Ono, M.; Miki, K.; Tobe, Y., Org. Lett. 2006, 8, 1197.

[31] Li, C.-K.; Zeng, Y.; Wang, J.-B. Tetrahedron Lett. 2009, 50, 2956.

[32] Ye, S.; Gao, K.; Zhou, H.; Yang, X.; Wu, J. Chem. Commun. 2009, 5406.

[33] (a) Ye, S.-Q.; Yang X.-D.; Wu, J. Chem. Commun. 2010, 46, 2950. (b) Ye, S.-Q.; Ren, H.; Wu, J. J. Comb. Chem. 2010, 12, 670.

[34] Bryan C. S.; Lautens, M. Org. Lett. 2010, 12, 2754.

[35] Kim, K. H.; Kim, S. H.; Park, B. R.; Kim, J.-N. Tetrahedron Lett. 2010, 51, 3368.

[36] Lim, C. H.; Kim, K. H.; Lim, J. W.; Kim, J. N. Tetrahedron Lett. 2013, 54, 5808 .

[37] (a) Wang, W.-Y.; Sun, L. L.; Deng, C. L.; Tang, R.-Y.; Zhang, X.-G. Synthesis 2013, 45, 118.

(b) Zhang, T.; Chen, F.; Zhang, X.-H.; Qian, P.-C.; Zhang, X.-G. J. Org. Chem. 2019, 84, 307.

[38] (a) Álvarez, E.; Miguel, D.; García-García, P.; FernándezRodríguez, M. A.; Rodríguez, F.; Sanz, R. Synthesis 2012, 44, 1874. (b) Alvarez, E.; Nieto Faza, O.; Silva Lopez, C.; Fernandez-Rodriguez, M. A.; Sanz, R. Chem.-Eur. J. 2015, 21, 12889.

[39] Chen, Z.; Jia, X.; Huang, J.; Yuan, J. J. Org. Chem. 2014, 79, 10674.

[40] Hou, Q. W.; Zhang, Z. H.; Kong, F. J.; Wang, S. Z.; Wang, H. Q.; Yao, Z. J. Chem. Commun. 2013, 49, 695.

[41] Aziz, J.; Brion, J.-D.; Alami, M.; Hamze, A. RSC Adv. 2015, 5, 74391.

[42] Shin, S.; Son, J. Y.; Choi, C.; Kim, S.; Lee, P. H. J. Org. Chem. 2016, 81, 11706 .

[43] Zhou, B.; Wu, Z.; Qi, W.; Sun, X.; Zhang, Y. Adv. Synth. Catal. 2018, 360, 4480.

[44] Peng, J.-B.; Wu, F.-P.; Spannenberg, A.; Wu, X.-F. Chem. Eur. J. 2019, 25, 8696.

[45] Borthakur, S.; Baruah, S.; Sarma, B.; Gogoi, S. Org. Lett. 2019, 21, 2768.

[46] (a) Hashmi, A. S. K.; Braun, I.; Noesel, P.; Schaedlich, J.; Wieteck, M.; Rudolph, M.; Rominger, F. Angew. Chem., Int. Ed. 2012, 51, 4456.

(b) Plajer, A; Ahrens, L.; Wieteck, M.; Lustosa, D. M.; Babaahmadi, R.; Yates, B.; Ariafard, A.; Rudolph, M.; Rominger, F.; Hashmi, A. S. K. Chem.-Eur. J. 2018, 24, 10766.

[47] Salacz, L.; Girard, N.; Suffert, J.; Blond, G. Molecules 2019, 24, 595.

[48] Whitlock, H. W.; Sandvick, P. E.; Overman, L. E.; Reichardt, P. B. J Org. Chem. 1969, 34, 879.

[49] (a) Schreiner, P. R.; Prall, M.; Lutz, V. Angew. Chem., Int. Ed. 2003, 42, 5757.

(b) Garcia-Garcia, P.; Sanjuan, A. M.; Rashid, M. A.; MartinezCuezva, A.; Fernandez-Rodriguez, M. A.; Rodriguez, F.; Sanz, R. J. Org. Chem. 2017, 82, 1155.

[50] Huang, X.; Yang, Y. Org. Lett. 2007, 9, 1667.

[51] Xiao, Q.; Zhu, H.; Li, G.; Chen, Z. Adv. Synth. Catal. 2014, 356, 3809.

[52] Martinelli, C.; Cardone, A.; Pinto, V.; Mastropasqua Talamo, M.; D'Arienzo, M. L.; Mesto, E.; Schingaro, E.; Scordari, F.; Naso, F.; 
Musio, R.; Farinola, G. M. Org. Lett. 2014, 16, 3424

[53] König, B.; Pitsch, W.; Klein, M.; Vasold, R.; Prall, M.; Schreiner, P. R. J. Org. Chem. 2001, 66, 1742 .

[54] Kovalenko, S. V.; Peabody, S.; Manoharan, M.; Clark, R. J.; Alabugin, I. V. Org. Lett. 2004, 6, 2457.

[55] Peabody, S. W.; Breiner, B.; Kovalenko, S. V.; Patil, S.; Alabugin, I. V. Org. Biomol. Chem. 2005, 3, 218

[56] Qin, X.-Y.; He, L.; Li, J.; Hao, W.-J.; Tu, S.-J.; Jiang, B. Chem.
Commun. 2019, 55, 3227.

[57] Cordier, P.; Aubert, C.; Malacria, M.; Lacote, E.; Gandon, V. Angew. Chem., Int. Ed. 2009, 48, 8757.

[58] Sousa, C. M.; Berthet, J.; Delbaere, S.; Coelho P. J. J. Org. Chem. 2014, 79, 5781.

[59] Sai, M.; Matsubara, S. Synlett 2014, 25, 2067.

[60] Warner, A. J.; Enright, K. M.; Cole, J. M.; Yuan, K.; McGough, J. S.; Ingleson, M. J. Org. Biomol. Chem. 2019, 17, 5520.

(Cheng, F.) 